\title{
Local Algebras of Observables and Pointlike Localized Fields
}

\author{
Klaus Fredenhagen ${ }^{1}$ and Joachim Hertel $^{2} \star^{\star}$ \\ 1 Fakultät für Physik, Universität Freiburg, D-7800 Freiburg i. Br., Federal Republic of Germany \\ 2 Nordseeklinik Kaiserhof, D-2972 Borkum, Federal Republic of Germany
}

\begin{abstract}
We present a method to recover Wightman fields from a HaagKastler theory of local observables. This may provide a basis for the comparison of different theories and for an algebraic description of high energy behaviour.
\end{abstract}

\section{Introduction}

For the discussion of structural properties of quantum field theory, it has turned out to be advantageous to use the framework of algebras of local observables [1]. In this framework one assigns to each bounded region $\mathcal{O}$ of space-time the $C^{*}$-algebra $\mathfrak{A}(\mathcal{O})$ which is generated by the observables measurable in $\mathcal{O}$. The "local net" $\mathcal{O} \rightarrow \mathfrak{U}(\mathcal{O})$ is subject to the requirements of locality and translation covariance, and according to Haag and Kastler [1], it contains all the physical information which can be obtained from the theory.

This point of view has been very successful in deriving those properties of a quantum field theory which are consequences of general principles, such as the existence of scattering states [2], and the structure of superselection sectors [3]. On the other hand, the algebraic point of view has not yet been equally useful for a discussion of particular properties of a given theory. This comes from the fact that it is difficult to construct the local net explicitly, and no effective notion of "similarity" for different local nets, no perturbative treatment of the algebraic structure has been developed.

We know that in field theory one can use the same set of fields to describe different theories, labeled by parameters like coupling constants and masses. Many properties of the theory are expressed with the aid of these fields. On the other hand, there are in general many other fields which can be used to describe the same theory. Roughly speaking, fields play a role similar to that of

* On leave of absence from II. Institut für Theoretische Physik, Hamburg, D-2000 Hamburg 50, Federal Republic of Germany 
a coordinate system in differential geometry, and because of the arbitrariness in the choice of a field, it is often unclear whether properties of the field are really intrinsic properties of the theory.

In this paper, we start from a local net $\mathcal{O} \rightarrow \mathfrak{A}(\mathcal{O})$, represented in a Hilbert space $\mathscr{H}$ such that the spectrum condition is fulfilled. Our purpose is the determination of all fields which are associated to this net. Hopefully, this set of fields can be used to obtain a more detailed description of the given local net, but this goes beyond the scope of the present paper.

Fields, being localized at a space-time point, should be, in a certain sense, limits of observables localized in a shrinking sequence of spacetime regions. Since the only bounded operators which are localized at a point are the multiples of the identity [4], fields must be singular objects. Physically, this may be understood as a consequence of the quantum mechanical uncertainty relation which says that measurements performed in a small region of spacetime require a large amount of energy-momentum transfer ${ }^{1}$. In a massive theory, one may suppose that this is the only reason for the unboundedness of local fields, so matrix elements of fields between state vectors with sufficiently good high energy behaviour should be finite [12].

For these reasons, we think it might be reasonable to restrict ourselves to Wightman fields $A(x)$ which are associated to the given net and fulfil higher order $H$-bounds of the form

$$
\left\|(1+H)^{-k} A(1+H)^{-k}\right\|<\infty
$$

for some $k>0, H$ being the energy operator. We shall show that these fields are characterized by the fact that $(1+H)^{-k} A(x)(1+H)^{-k}$ belongs to the weak closure of $(1+H)^{-k} \mathfrak{A}(\mathcal{O})(1+H)^{-k}$ for any neighbourhood $\mathcal{O}$ of $x$. Thus at least the set of Wightman fields with polynomially bounded high energy behaviour is simply related to the underlying local net.

\section{The Associated Fields of a Local Net}

Our starting point is a local net $\mathcal{O} \rightarrow \mathfrak{A}(\mathcal{O})$ where to each bounded space-time region $\mathcal{O}$ there is assigned a von-Neumann-algebra $\mathfrak{A}(\mathcal{O})$ such that $\mathcal{O}_{1} \subset \mathcal{O}_{2}$ implies $\mathfrak{A}\left(\mathcal{O}_{1}\right) \subset \mathfrak{A}\left(\mathcal{O}_{2}\right)$. We assume that all these algebras are represented in some Hilbert space $\mathscr{H}$ such that the following conditions hold:

(A1) Locality: If the region $\mathcal{O}_{1}$ is contained in the spacelike complement $\mathcal{O}_{2}^{\prime}$ of some region $\mathcal{O}_{2}$, then the corresponding algebras commute:

$$
\mathfrak{A}\left(\mathcal{O}_{1}\right) \subset \mathfrak{A}\left(\mathcal{O}_{2}\right)^{\prime} .
$$

(A2) Translation covariance: There is a strongly continuous unitary representation $x \rightarrow U(x)$ of the translation group in $\mathscr{H}$ such that

$$
U(x) \mathfrak{A}(\mathcal{O}) U(-x) \subset \mathfrak{A}(\mathcal{O}+x) .
$$

1 See the discussion of Gedankenexperiments in Bohr, N., Rosenfeld, L.: Dan. Mat. Fys. Madd XII 1933, Phys. Rev. 78, 794 (1950) 
(A3) Spectrum condition: The joint spectrum of the generators $P_{\mu}$ of $U, \mu$ $=0, \ldots, 3$ is contained in the closed forward light cone $\bar{V}_{+}=\left\{p \in \mathbb{R}^{4} \mid p_{0} \geqq 0\right.$, $\left.p^{2} \geqq 0\right\}$.

The class $F$ of Wightman fields which we want to consider is characterized by two conditions, a regularity condition (F1) and a locality condition (F2). Let us first discuss the regularity condition!

Let $H=P_{0}$ be the energy operator in $\mathscr{H}$. According to the spectrum condition (A3),

$$
R=(1+H)^{-1}
$$

is a positive bounded operator with norm smaller than one. Then we can formulate our regularity condition:

(F1) Every $A \in F, A \equiv A(0)$, is a qudratic form on $\mathscr{C}^{\infty}(H)=\bigcap_{k>0} R^{k} \mathscr{H}$ such that $R^{k} A R^{k}$ is bounded for some $k>0$.

Usually, Wightman fields are defined as operator-valued tempered distributions with a common invariant dense domain of definition. But this is, in fact, a consequence of (F1) [12]:

2.1. Lemma. Let $A$ be a quadratic form which fulfils (F1) for some $k>0$. For $f \in \mathscr{S}\left(\mathbb{R}^{4}\right)$, set

$$
A(f)=\int d^{4} x f(x) U(x) A U(-x) .
$$

Then $R^{k-\ell} A(f) R^{k+\ell}$ is bounded for any $\ell \in \mathbb{R}$.

Proof. Clearly $R^{k} A(f) R^{k}$ is bounded for any $f \in \mathscr{S}\left(\mathbb{R}^{4}\right)$. By repeated use of the identity

$$
R A(f)-A(f) R=R A\left(\partial_{t} f\right) R
$$

we get the lemma at least for integer $\ell$. The result for noninteger values of $\ell$ then follows from interpolation arguments [5, Appendix to IX.4]. q.e.d.

So $\left\|R^{k} A R^{k}\right\|<\infty$ implies $\left\|A(f) R^{2 k}\right\|<\infty$, hence there is a unique operator on $\mathscr{C}^{\infty}(H)$ which coincides with $A(f)$ as a quadratic form and which will be denoted by the same symbol. $\mathscr{C}^{\infty}(H)$ is an invariant domain of definition for all operators $A(f)$, where $A$ satisfies (F1) and $f \in \mathscr{S}\left(\mathbb{R}^{4}\right)$. Denoting the adjoint quadratic form by $A^{*}$, we get immediately $A^{*}(f) \subset A(f)^{*}$, so $A(f)$ is closable, and the set of fields has a hermitean structure. Finally, for $\Phi, \Psi \in \mathscr{C}^{\infty}(H)$

$$
|(\Phi, A(f) \Psi)| \leqq\left|\left(\Phi,(1+H)^{2 k} \Psi\right)\right|\left\|R^{k} A R^{k}\right\| \int|f| d^{4} x
$$

so the temperedness condition is automatically fulfilled.

On the other hand, let $A$ be an operator-valued distribution with $\mathscr{C}^{\infty}(H)$ as invariant domain such that for some $k>0$

$$
\left\|R^{k} A(f) R^{k}\right\| \leqq\|f\|_{\mathscr{S}},
$$


with $f \in \mathscr{S}\left(\mathbb{R}^{4}\right)$ and $\|\cdot\|_{\mathscr{S}}$ denoting some $\mathscr{S}$-norm. Then there is a quadratic form $A_{0}$ on $\mathscr{C}^{\infty}(H)$ which satisfies (F1) for some $k^{\prime} \geqq k$ such that

$$
A_{0}(f)=A(f) \text {. }
$$

This may be seen as follows: Since the norm of $R^{k} A(f) R^{k}$ is translation invariant, the $\mathscr{S}$-norm $\|\cdot\|_{\mathscr{S}}$ can be chosen to be of the form

$$
\|f\|_{\mathscr{S}}=\text { const } \int d^{4} x \sum_{|\alpha| \leqq \ell}\left|\left(\partial^{\alpha} f\right)(x)\right|
$$
where $\ell \in \mathbb{N}, \alpha=\left(\alpha_{0}, \ldots, \alpha_{3}\right) \in \mathbb{Z}_{+}^{4},|\alpha|=\sum_{\mu=0}^{3} \alpha_{\mu}$ and $\partial^{\alpha}=\partial_{0}^{\alpha_{0}} \cdot \partial_{3}^{\alpha_{3}}$. As $\left\|R P_{\mu}\right\| \leqq 1$ and
$\left[P_{\mu}, A(f)\right]=A\left(\partial_{\mu} f\right)$, we have

$$
\left\|R^{k+|\alpha|} A\left(\partial^{\alpha} f\right) R^{k+|\alpha|}\right\| \leqq 2^{|\alpha|}\|f\|_{\mathscr{S}}
$$

Let $\Delta$ be the Laplacian on $\mathbb{R}^{4}$. Then from (2.9) and (2.8)

$$
\left\|R^{k+2 \ell} A\left((1-\Delta)^{\ell} f\right) R^{k+2 \ell}\right\| \leqq \text { const } \int d^{4} x \sum_{|\alpha| \leqq \ell}\left|\left(\partial^{\alpha} f\right)(x)\right|,
$$

which implies

$$
\left\|R^{k+2 \ell} A(g) R^{k+2 \ell}\right\| \leqq \text { const } \int d^{4} x|g(x)|
$$

for any $g \in \mathscr{S}\left(\mathbb{R}^{4}\right)$, since $(1-\Delta)$ is invertible on $\mathscr{S}\left(\mathbb{R}^{4}\right)$ and $\partial^{\alpha}(1-\Delta)^{-\ell}$ is a bounded operator on $\mathscr{L}^{1}\left(\mathbb{R}^{4}\right)$ for any $\alpha$ with $|\alpha| \leqq \ell$.

Now choose $f \in \mathscr{S}\left(\mathbb{R}^{4}\right)$ with $\int f=1$ and set $f_{n}(x)=n^{4} f(n x)$. Then the uniformly bounded sequence $\left(R^{k+2 \ell} A\left(f_{n}\right) R^{k+2 \ell}\right)_{n \in \mathbb{N}}$ converges weakly to some $B$ $\in \mathscr{B}(\mathscr{H})$ due to the strong continuity of the translation group representation. With

$$
A_{0}:=(1+H)^{k+2 \ell} B(1+H)^{k+2 \ell},
$$

we get a quadratic form on $\mathscr{C}^{\infty}(H)$ with the desired properties.

To finish our discussion of the regularity condition (F1), we mention that bounds of the form (2.6) are known to hold for members of the Borchers class of the free massive field and for interacting fields in the superrenormalizable theories which have been constructed so far. The bound is violated by the free massless field. This may support our conjecture that for massive theories (F1) holds in typical cases.

We now want to specify how the Wightman fields of the class $F$ are related to the local net. We propose the following locality condition:

(F2) Let $A$ be a quadratic form which satisfies (F1). $A$ is contained in $F$ if for any neighbourhood $\mathcal{O}$ of the origin and any $C \in \mathfrak{U}(\mathcal{O})^{\prime}$ such that $C \mathscr{C}^{\infty}(H) \subset \mathscr{C}^{\infty}(H)$ and $C^{*} \mathscr{C}^{\infty}(H) \subset \mathscr{C}^{\infty}(H)$

$$
C A=A C
$$

holds in the sense of quadratic forms on $\mathscr{C}^{\infty}(H)$. 
This condition might seem to be very weak, but in fact it implies that the minimal closed extensions $A(f)^{-}$of the operators $A(f)$ are affiliated to the local v. Neumann-algebras:

2.2. Lemma. Let $A \in F$ and $f \in \mathscr{S}\left(\mathbb{R}^{4}\right)$ with supp $f \subset \mathcal{O}$ for some open bounded region $\mathcal{O}$. Then $A(f)^{-}$is affiliated to $\mathfrak{U}(\mathcal{O})$, i.e. $A(f)^{-} \eta \mathfrak{U}(\mathcal{O})$.

Proof. We have to show that $\Psi \in \mathscr{D}\left(A(f)^{-}\right), C \in \mathfrak{A}(\mathcal{O})^{\prime}$ implies

$$
\begin{aligned}
& C \Psi \in \mathscr{D}\left(A(f)^{-}\right) \\
& C A(f)^{-} \Psi=A(f)^{-} C \Psi .
\end{aligned}
$$

Let $\Psi \in \mathscr{D}\left(A(f)^{-}\right)$. Then there exists a sequence $\Psi_{n} \in \mathscr{C}^{\infty}(H)$ such that $\Psi_{n} \longrightarrow \Psi$ and $A(f) \Psi_{n} \longrightarrow A(f)^{-} \Psi$. As $\mathcal{O}$ is bounded, supp $f$ is compact, so there are open neighbourhoods $\mathcal{O}_{1}$ and $\mathcal{O}_{2}$ of the origin such that

$$
\operatorname{supp} f+\mathcal{O}_{1}+\mathcal{O}_{2} \subset \mathcal{O} \text {. }
$$

Let $h \in \mathscr{S}\left(\mathbb{R}^{4}\right)$ with supp $h \subset \mathcal{O}_{2}, \int h=1$, and set

$$
C_{n}:=n^{4} \int d^{4} x h(n x) U(x) C U(-x), \quad n \in \mathbb{N} .
$$

Then $C_{n} \in \mathfrak{A}\left(\operatorname{supp} f+\mathcal{O}_{1}\right)^{\prime}, C_{n} \mathscr{C}^{\infty}(H) \subset \mathscr{C}^{\infty}(H), C_{n}^{*} \mathscr{C}^{\infty}(H) \subset \mathscr{C}^{\infty}(H), C_{n} \longrightarrow C$ and $\left\|C_{n}\right\| \leqq\|C\|$. Hence $C_{n} \Psi_{n} \underset{s}{\longrightarrow} C \Psi$ and from $(\mathrm{F} 2)$

$$
A(f) C_{n} \Psi_{n}=C_{n} A(f) \Psi_{n} \underset{s}{\longrightarrow} C A(f)^{-} \Psi .
$$

This proves (2.14). q.e.d.

It is well known that $A(f)^{-} \eta \mathfrak{U}(\mathcal{O})$ also implies that $A(f)^{*} \eta \mathfrak{U}(\mathcal{O})$ [13]. So $A(f)^{*}$ is a local closed extension of $A^{*}(f)^{-}$. As a consequence of Lemma 2.2 and from locality of the underlying net, it can be easily shown that all Wightman fields in the class $F$ are local and relatively local.

We now can state our main result which is more or less obvious at this stage of the discussion.

Theorem. A quadratic form $A$ on $\mathscr{C}^{\infty}(H)$ is contained in $F$ if and only if there exists a $k>0$ such that $R^{k} A R^{k}$ is contained in the weak closure of $R^{k} \mathfrak{U}(\mathcal{O}) R^{k}$ for any neighgbourhood $\mathcal{O}$ of the origin.

Proof. a) Let $A \in F$ such that $R^{k} A R^{k}$ is bounded, and let $\mathcal{O}$ be an open neighbourhood of the origin. Let $f \in \mathscr{S}\left(\mathbb{R}^{4}\right)$ with supp $f \subset \mathcal{O}$. From Lemma 2.2 we know that $A(f)^{-}$is affiliated to $\mathfrak{A}(\mathcal{O})$. Let

$$
A(f)^{-}=V|A(f)|=\left|A(f)^{*}\right| V
$$

be the left, respectively right, polar decomposition of $A(f)^{-}$. Then the partial isometry $V$ and the spectral projections of the positive operators $|A(f)|$ and $\left|A(f)^{*}\right|$ are contained in $\mathfrak{A}(\mathcal{O})[6]$. The initial support of $V$ is the range of $|A(f)|$, the final support is the range of $\left|A(f)^{*}\right|$. So

$$
A(f)^{-}=V|A(f)|^{\frac{1}{2}}|A(f)|^{\frac{1}{2}}=\left|A(f)^{*}\right|^{\frac{1}{2}} V|A(f)|^{\frac{1}{2}} .
$$


Let $E_{\lambda}, \lambda>0$, be the spectral projection of $|A(f)|$ on the interval $[0, \lambda]$. Then $T_{\lambda}=\left|A(f)^{*}\right|^{\frac{1}{2}} V E_{\lambda}|A(f)|^{\frac{1}{2}} \in \mathfrak{U}(\mathcal{O})$. Since the bounds $\left\|A(f) R^{2 k}\right\|,\left\|A(f)^{*} R^{2 k}\right\|<\infty$ imply the bounds $\left\||A(f)|^{\frac{1}{2}} R^{k}\right\|, \| R^{k} \mid A\left(\left.f^{*}\right|^{\frac{1}{2}} \|<\infty\right.$ [7, VI, 1.38], we have

$$
R^{k} A(f) R^{k}=w-\lim _{\lambda \rightarrow \infty} R^{k} T_{\lambda} R^{k} .
$$

So $R^{k} A(f) R^{k}$ is in the weak closure of $R^{k} \mathfrak{A}(\mathcal{O}) R^{k}$ for any $f \in \mathscr{S}\left(\mathbb{R}^{4}\right)$ with $\operatorname{supp} f \subset \mathcal{O}$. Now as before choose $f$ with $\int f=1$ and set $f_{n}(x)=n^{4} f(n x)$. Then from the strong continuity of the translations we have

$$
R^{k} A\left(f_{n}\right) R^{k} \underset{w}{\longrightarrow} R^{k} A R^{k},
$$

which proves one direction of the theorem.

b) To prove the other direction, we consider a quadratic form $A$ on $\mathscr{C}^{\infty}(H)$ such that $R^{k} A R^{k}$ is in the weak closure of $R^{k} \mathfrak{Q}(\mathcal{O}) R^{k}$ for any neighbourhood $\mathcal{O}$ of the origin. Let $\mathcal{O}$ be such a neighbourhood, and let $C \in \mathfrak{U}(\mathcal{O})$ with $C \mathscr{C}^{\infty}(H)$, $C^{*} \mathscr{C}^{\infty}(H) \subset \mathscr{C}^{\infty}(H)$. There is a net $A_{\lambda} \in \mathfrak{U}(\mathcal{O})$ such that $R^{k} A_{\lambda} R^{k} \underset{w}{\longrightarrow} R^{k} A R^{k}$. Then for any $\Phi, \Psi \in \mathscr{C}^{\infty}(H)$,

$$
(\Phi, C A \Psi)=\lim _{\lambda}\left(\Phi, C A_{\lambda} \Psi\right)=\lim _{\lambda}\left(\Phi, A_{\lambda} C \Psi\right)=(\Phi, A C \Psi)
$$

hence $A$ fulfills the locality condition (F2). q.e.d.

\section{Discussion of the Results}

Given a local net $\mathcal{O} \rightarrow \mathfrak{U}(\mathcal{O})$ and a covariant representation $(\pi, \mathscr{H})$ with spectrum condition, we have found a class of Wightman fields which is intrinsically characterized by the local net and the representation. Of course one should expect that this class should be the same for each locally normal representation. At the moment we are unable to prove this conjecture except for representations which are particle excitations of some vacuum representation [8].

More interesting is the question: which information of the theory is coded into the structure of the field space $F$ ? $F$ has the structure of an inductive limit of Banach spaces $F_{k}, k \geqq 0$, where

$$
F_{k}=\left\{A \in F \mid\left\|R^{k} A R^{k}\right\|<\infty\right\} .
$$

$d(A)=\inf \left\{k \geqq 0 \mid A \in F_{k}\right\}$ may be called the high energy dimension of the field $A$. In typical cases one should expect that there is only a finite number of linearly independent fields in each space $F_{k}$. In these cases, theories may be partially classified by their dimension function $k \rightarrow \operatorname{dim} F_{k}$. So, asymptotically free theories probably have the same dimension function as the associated free theory. Another interesting aspect of the inductive limit structure of $F$ is the existence of a set of subnets $\mathcal{O} \rightarrow \mathfrak{A}_{k}(\mathcal{O})$, where $\mathfrak{A}_{k}(\mathcal{O})$ is generated by the bounded functions of $A(f)$ with $A \in F_{k}$ and $\operatorname{supp} f \subset \mathcal{O}$. From the experience with free theories and perturbation theory, one expects that $\mathfrak{A}_{k}=\mathfrak{U}$ for some $k>0$. If a local net is generated by its associated Wightman fields, properties like weak additivity and duality may be obtained directly. 
So there is a certain amount of information in the field space $F$ which may be used to specify a given theory to some degree. To get a more detailed description, one could try to identify a product structure in $F$ induced by the product structure in $\mathfrak{A}$. Such so-called operator product expansions are known for free fields and in conformal field theory [9], and they presumably hold in any renormalizable field theory. Operator product expansions may lead to the definition of a theory without referring to a Lagrangian [10]. Perhaps our methods may serve to clarify the intrinsic role of these expansions; we hope to discuss these questions elsewhere.

Acknowledgements. This paper is partially based on one of the author's (J.H.) thesis [11]. We are indebted to Professor Rudolf Haag for posing the problem and for many helpful discussions. One of us (J.H.) also wants to thank D. Buchholz for discussions.

\section{References}

1. Haag, R., Kastler, D.: J. Math. Phys. 5, 848-261 (1964)

2. Haag, R.: Phys. Rev. 112, 669 (1958)

Ruelle, D.: Helv. Phys. Acta 35, 147 (1962)

3. Doplicher, S., Haag, R., Roberts, J.: Commun. Math. Phys. 23, 199-230 and 35, 49-85 (1974)

4. Wightman, A.S.: Ann. Inst. Poincaré, 1A, 403-420 (1964)

5. Reed, M., Simon, B.: Methods of modern mathematical physics. II. New York: Academic Press, Inc. 1975

6. Bratteli, O., Robinson, D.W.: Operator algebras and quantum statistical mechanics. I. New York: Springer 1979

7. Kato, T.: Perturbation theory for linear operators. Berlin, Heidelberg, New York: Springer 1966

8. Buchholz, D., Fredenhagen, K.: Locality and the structure of particle states in relativistic quantum field theory (to be published)

9. Mack, G.: Commun. Math. Phys. 53, 155-184 (1977)

10. Wilson, K.: Phys. Rev. 179, 1499 (1969)

11. Hertel, J.: Lokale Quantentheorie und Felder am Punkt. Thesis, Hamburg 1980

12. Haag, R.: Ann. Phys. 11, 29-34 (1963)

13. Dixmièr, J.: Les algebrès d'operateurs. Paris: Gauthier-Villars 1969

Communicated by R. Haag

Received April 2, 1981 
